\title{
Programas bilingües en centros de Educación Primaria, ¿qué dicen los docentes implicados en su desarrollo?
}

\section{Bilingual programs in Primary Education: What do teachers say?}

\author{
Mónica Porto Currás ${ }^{1}$ \\ monicapc@um.es \\ Ma José Bolarín Martinez \\ mbolarin@um.es \\ María Lova Mellado \\ maria88_trebol@hotmail.com \\ Universidad de Murcia, España
}

\begin{abstract}
Resumen:
INTRODUCCIÓN. Se presentan en este artículo valoraciones de docentes implicados en las primeras promociones de los Programas Bilingües en Centros de Educación Primaria de la Región de Murcia, su satisfacción con su desarrollo y las propuestas de mejoras que consideran para su continuidad en todos los centros educativos. MÉTODO. Con un enfoque de investigación descriptivo, y siguiendo un muestreo estratificado, se entrevistó a 78 docentes de centros públicos y privados, distribuidos entre todas las comarcas de la Región y con diversos años de experiencia. RESULTADOS Y DISCUSIÓN. Los principales resultados indican un alto grado de satisfacción profesional, a pesar de las quejas generalizadas por la poca implicación de la Administración educativa y dudas sobre las ventajas de estos Pro-
\end{abstract}

\begin{abstract}
:
INTRODUCTION. This article explores the satisfaction of teachers in the Region of Murcia involved in bilingual education and presents the proposals they make to improve its implementation. METHOD. With a descriptive research methodology, 78 teachers of public and private primary schools distributed among all areas of the Region of Murcia and with several years of experience in bilingual education were interviewed. RESULTS AND DISCUSSION The main results show that the satisfaction of teachers is high, despite widespread complaints about the lack of involvement of the education authorities and doubts about the advantages of these programs for students with educational needs.
\end{abstract}

1 Dirección para correspondencia (correspondence address):

Mónica Porto Curras. Universidad de Murcia. Departamento de Didáctica y Organización Escolar. Facultad de Educación. Campus de Espinardo, s/n. 30100 Murcia (España). 
gramas para alumnado con necesidades de apoyo educativo.

\section{Palabras Clave:}

Enseñanza bilingüe; perspectiva docente; satisfacción profesional; propuestas de mejora.

\section{Key words:}

Bilingual education; teaching perspective; professional satisfaction; improvement proposals.

\section{Résumé :}

INTRODUCTION. Cet article présente les évaluations des enseignants impliqués dans les premières promotions des programmes bilingues dans les centres d'enseignement primaire de la région de Murcie, leur satisfaction quant à leur développement et les propositions d'améliorations envisagées pour leur continuité dans tous les centres éducatifs. MÉTHODE. Dans le cadre d'une démarche de recherche descriptive et d'un échantillonnage stratifié, 78 enseignants de centres publics et privés ont été interrogés, répartis dans cantons de la région et avec plusieurs années d'expérience. RÉSULTATS ET DISCUSSION. Les principaux résultats indiquent un degré élevé de satisfaction professionnelle, malgré les nombreuses plaintes concernant le manque d'implication de l'administration de l'éducation et des doutes quant aux avantages de ces programmes pour les étudiants ayant des besoins en matière de soutien éducatif.

\section{Mots clés:}

Éducation bilingue; perspective pédagogique; satisfaction professionnelle; propositions d'amélioration.

Fecha de recepción: 29-03-2019

Fecha de aceptación: 27-05-2019

\section{Introducción}

El gobierno español, igual que otros países europeos, ha incorporado progresivamente recomendaciones específicas en materia de lenguas con el fin de fomentar el aprendizaje de, al menos, dos lenguas extranjeras. Así, la Ley Orgánica de Educación, del 3 de mayo de 2006 (LOE) señala la necesidad de capacitar al alumnado para la comunicación en la lengua oficial y cooficial, si la hubiere, y en una o más lenguas extranjeras (artículo 2.j) y propone que las Administraciones educativas promuevan la formación en lenguas extranjeras de todo el profesorado, independientemente de su especialidad, estableciendo programas específicos de formación en este ámbito (artículo 102.3).

En el preámbulo de la actual Ley Orgánica 8/2013, de 9 de diciembre, para la Mejora de la Calidad Educativa (LOMCE), se destaca que la inclusión de una segunda lengua extranjera o, incluso, una tercera se ha convertido en una prioridad en la educación, como respuesta al 
proceso de globalización social y como objetivo para la construcción de un proyecto europeo. En este sentido se apoya el plurilingüismo, y se apuesta por redoblar esfuerzos para favorecer los niveles de expresión y comprensión que permitan a los estudiantes responder a las demandas de una sociedad cambiante.

Si bien todas las Comunidades Autónomas de España han introducido medidas ambiciosas en materia educativa, la Región de Murcia pretende incorporar la enseñanza bilingüe español-inglés en todos los centros docentes de Educación Infantil y Primaria, públicos y privados, buscando un uso adecuado y simultáneo de la lengua castellana y de la lengua inglesa de los estudiantes en Educación Primaria, así como la adquisición de la competencia comunicativa suficiente para desenvolverse con naturalidad en situaciones cotidianas.

Este artículo se centra en la adopción del bilingüismo en los centros de Educación Primaria de la Región de Murcia y, se presenta una valoración de docentes implicados en las primeras promociones de los Programas Bilingües en esta Comunidad Autónoma, su satisfacción con su desarrollo y las propuestas de mejoras que consideran necesarias para su continuidad en todos los centros educativos de Educación Primaria.

\section{Antecedentes: programas bilingües en la Comunidad Autónoma de la Región de Murcia}

Los denominados Proyectos curriculares integrados de enseñanza bilingüe, en este caso en la modalidad español-inglés en la Comunidad Autónoma de la Región de Murcia, se inician al amparo del acuerdo de colaboración suscrito entre el Ministerio de Educación y el British Council, por el que se implantaba en colegios de Educación Primaria un currículo bilingüe consistente en el $40 \%$ de las horas lectivas en inglés y orientado a la obtención simultánea de los títulos académicos de los dos países, en el ámbito de la educación obligatoria, siendo el MEC y el British Council encargados de formar al profesorado.

En este marco, la Consejería de Educación, Formación y Empleo, basándose en el Decreto 286/2007 de la Región, vigente en ese momento, que establecía como uno de los objetivos principales en la Etapa de Educación Primaria, la "adquisición en al menos una lengua extranjera, de la competencia comunicativa básica que les permita expresar y com- 
prender mensajes sencillos y desenvolverse en situaciones cotidianas" (artículo 4.f) y convencida de la necesidad de inculcar a los futuros adolescentes la importancia de hablar y escribir adecuadamente la lengua inglesa, puso en marcha la implantación del programa colegios bilingües Región de Murcia (CBM) en 25 centros, 23 pertenecientes al sector público y 2 al privado-concertado, de Educación Infantil y Primaria de la Región a través de la Orden de 25 de mayo de 2009. Dicha orden regulaba la enseñanza bilingüe español-inglés para centros docentes de Educación Infantil y Primaria, establecía el Programa Colegios Bilingües Región de Murcia y, en el artículo 1 dispone que "se entiende por la enseñanza bilingüe la que permite impartir, al menos, un tercio del horario lectivo semanal en lengua inglesa. Para ello, los centros seleccionados, además del horario dedicado a Idioma Extranjero: Inglés, deberán impartir también en Inglés el resto del horario semanal hasta el mencionado tercio de otras áreas. Dicha elección podrá ser llevada a cabo entre las siguientes áreas: Conocimiento del Medio Natural, Social y Cultural, Educación Artística y Educación Física".

Para ello, contempla la asignación de medidas específicas de apoyo, entre las que cabe destacar: orientaciones didácticas, ampliación del personal del centro mediante Auxiliares de Conversación de lengua inglesa, que contribuirán favorablemente al desarrollo del programa, la participación del coordinador y del profesorado de asignaturas no lingüísticas (al menos durante un curso escolar completo, se valorará como mérito), percepción del complemento que se determine reglamentariamente y, plan de formación para el que dependiendo de la titulación aportada se plantearán dos tipos de programas formativos en idiomas:

- Maestros con titulación conocimientos y capacitación correspondiente a una titulación superior formación en países de habla inglesa durante dos semanas (certificación de 100 horas);

- Maestros habilitados en idioma extranjero: formación de cuatro semanas, en horario lectivo, en la propia Comunidad Autónoma (certificación de 100 horas) y, formación en países de habla inglesa durante dos semanas. (certificación de 100 horas).

Para los centros concertados la capacitación en países de habla en inglesa "correrá a cargo de de los titulares de los centros concertados" (artículo 12).

Tras dos años de implantación del Programa Colegios Bilingües Re- 
gión de Murcia (2009/2010 y 2010/2011), la Consejería de Educación, Formación y Empleo consideró necesario aprobar la Orden de 18 de abril de 2011, donde precisa que este programa pretende, el logro de un uso adecuado y simultáneo de la lengua castellana y de la lengua inglesa en Educación Primaria.

Posteriormente, la Comunidad Autónoma de esta Región, como consecuencia de la entrada en vigor de la LOMCE, ley Orgánica 8/2013, de 9 de diciembre, para la Mejora de la Calidad Educativa y con el fin de dar continuidad a este programa de enseñanza bilingüe y adaptarlo a la nueva ordenación educativa, decidió implantar un nuevo sistema de enseñanza bilingüe. Este programa está recogido en la Resolución 2 de junio de 2014, de la Dirección General de Calidad Educativa, Innovación y Atención a la Diversidad, por la que se define el Programa Sistema de Enseñanza Bilingüe Español-Inglés en Educación Primaria. Este nuevo programa, denominado Sistema de Enseñanza Bilingüe Español Inglés, permitió la posibilidad de adaptación del bilingüismo a la realidad de cada centro educativo. Para ello establece varias modalidades de inmersión lingüística:

a) Básica, en la que además del horario dedicado a la materia de Primera Lengua Extranjera, se impartirán en dicha lengua entre 1 y 2 horas semanales en cada curso.

b) Intermedia, en la que además del horario dedicado a la materia de Primera Lengua Extranjera, se impartirán en dicha lengua entre 2,5 y 4,5 horas semanales en cada curso. c) Avanzada, en la que además del horario dedicado a la materia de Primera Lengua Extranjera, se impartirán en dicha lengua 5 o más horas semanales en cada curso.

En el artículo 3 precisa que se podrá impartir todas las áreas utilizando el inglés como lengua vehicular, a excepción de las áreas de Lengua castellana y literatura, Lectura comprensiva, Profundización en lengua castellana y literatura, Segunda Lengua Extranjera, Lengua de signos española, Español Lengua Extranjera y Refuerzo en competencia en comunicación lingüística.

Es evidente que la enseñanza bilingüe ha supuesto un nuevo reto educativo para los docentes participantes en estos Programas, pues impartir materia de contenido por medio de una lengua extranjera no es lo mismo que impartirla por medio de la lengua materna (Halbach, 2008). 
Por ello, se ha considerado relevante conocer las valoraciones de los docentes implicados en esta enseñanza y sus recomendaciones para conseguir el objetivo marcado por esta Comunidad Autónoma de ampliar la implementación de este Programa a todos los centros educativos de Educación Primaria de la Región.

\section{Metodología}

De acuerdo con lo expuesto en los apartados anteriores, el objetivo general que se persiguen en este estudio se centra en conocer las valoraciones de los docentes responsables de impartir los Programas Bilingües en centros de Educación Primaria de la Región de Murcia sobre el funcionamiento de los mismos: satisfacción con la docencia impartida y los resultados conseguidos, así como sobre la implicación de la Consejería de Educación para su puesta en marcha.

Dada la naturaleza de la información que se buscaba, y para acercarnos a la perspectiva del docente, se optó por una metodología de investigación "no experimental descriptiva" (Salkind, 2009), ya que se trata de aportar una panorámica sobre la implementación de los Programas Bilingües en la Región de Murcia.

\subsection{Instrumento de recogida de información}

Para recoger la información se optó por la técnica de la entrevista, ya que se pretende conocer el objeto de estudio de esta investigación desde el punto de vista de los sujetos participantes, a través de una interacción con los mismos en la cual revelen sus pensamientos (Kvale, 2011). Para ello se diseñó una entrevista con un nivel de directividad medio-bajo, formulando preguntas relevantes a los profesores con la finalidad de obtener la información necesaria sobre el desarrollo de los Programas Bilingües; y semiestructurada en cuanto a que se plantean unos interrogantes de carácter abierto, con el objeto de que expresen libremente sus ideas, creencias, opiniones, valoraciones (Tójar, 2006).

La entrevista se organizó en dos grandes categorías: una primera categoría de clasificación, destinada a conocer el perfil de los docentes adscritos al Programa Bilingüe, y una segunda categoría organizada en preguntas abiertas relacionadas con la forma en que se concreta la ense- 
ñanza en estos Programas, la satisfacción de los docentes con su participación en los mismos y la implicación de la Consejería de Educación, la valoración de los resultados que se están obteniendo, así como las posibles propuestas de mejora que sugieren para optimizar estos Programas.

\subsection{Participantes}

Centrándonos en los participantes de este estudio, la selección se realizó atendiendo a un muestreo estratificado. Con la pretensión de tener representados docentes de centros públicos y privados de Educación Primaria de toda la Región de Murcia, se aplicaron criterios específicos de inclusión a la muestra tales como: comarca en la que ese sitúa el centro, titularidad del mismo y años de participación en el programa bilingüe.

De esta forma, el territorio se dividió en comarcas (de acuerdo con el criterio seguido por Atlas Global de la Región de Murcia, 2007) de comarcalización de la Región de Murcia. Atendiendo a las indicaciones ofrecidas por un experto en análisis metodológicos (Profesor Catedrático de Universidad de Murcia adscrito al Departamento de Psicología Básica y Metodología), y con la pretensión de obtener la máxima riqueza posible de información, se adoptó la decisión de seleccionar 24 centros públicos (22\% del total) y 6 centros privados ( $18 \%$ del total) distribuidos tal y como se expone en latabla1. Con estos criterios, pasaron a formar parte de la muestra productora de datos un total de 30 centros $(21 \%$ del total) y 78 docentes.

Tabla 1

Número de centros seleccionados de cada comarca

\begin{tabular}{lccccccccc}
\hline \multirow{2}{*}{ Comarca } & \multicolumn{3}{c}{$\begin{array}{c}\text { Centros } \\
\text { Públicos }\end{array}$} & \multicolumn{3}{c}{$\begin{array}{c}\text { Centros } \\
\text { Privados }\end{array}$} & \multicolumn{2}{c}{$\begin{array}{c}\text { Centros } \\
\text { Muestra }\end{array}$} \\
\cline { 2 - 11 } & Total & $\begin{array}{c}\text { Partici- } \\
\text { pantes }\end{array}$ & Total & $\begin{array}{c}\text { Partici- } \\
\text { pantes }\end{array}$ & $\begin{array}{c}\text { Partici- } \\
\text { pantes }\end{array}$ \\
\hline Área metropolitana de Murcia & 53 & 11 & 21 & 22 & 5 & 23 & 16 & 53 \\
\hline Campo de Cartagena-Mar Menor & 19 & 4 & 21 & 8 & 1 & 13 & 5 & 17 \\
\hline Bajo Guadalentín & 7 & 2 & 21 & 1 & 0 & 0 & 2 & 7 \\
\hline Noroeste & 9 & 2 & 22 & 0 & 0 & 0 & 2 & 7 \\
\hline Vega del Segura & 10 & 2 & 20 & 1 & 0 & 0 & 2 & 7 \\
\hline Altiplano de Jumilla & 4 & 1 & 25 & 0 & 0 & 0 & 1 & 3 \\
\hline Comarca de Lorca & 4 & 1 & 25 & 1 & 0 & 0 & 1 & 3 \\
\hline Cuenca de Mula & 2 & 1 & 20 & 1 & 0 & 0 & 1 & 3 \\
\hline Total & 108 & 24 & & 34 & 6 & & 30 & 100 \\
\hline
\end{tabular}


En la selección de centros educativos se priorizaron los años de experiencia en el programa bilingüe: diez centros llevan cinco años implicados en este programa, siete centros llevan cuatro años, nueve centros llevan tres años y cuatro centros llevan dos años. Asimismo, se ha buscado tener representación de centros con diferencia experiencia y en porcentajes similares entre centros públicos y privados.

En relación al profesorado entrevistado, el $80 \%$ son mujeres mientras que el $20 \%$ de participantes son hombres. Sin embargo, si atendemos a su distribución en función de la titularidad de los centros, vemos como en los centros privados la diferencia entre número de mujeres y de hombres son relativamente bajas ( $59 \%$ y $41 \%$ respectivamente), mientras que en los centros públicos la feminización de la enseñanza es mucho más visible, siendo el $85 \%$ de la muestra en estos centros mujeres.

Respecto a la edad, en la Tabla 2 se puede observar como la mayoría se encuentra en la franja comprendida entre los 31 y 40 años, si bien, en los centros privados todo el profesorado de la muestra es menor de 50 años y el porcentaje de maestros menores de 31 años es muy elevado (35\%). En los centros públicos, en cambio, es mayor el porcentaje de profesores que se encuentran en la franja de 41 a 50 años (31\% frente al $12 \%$ de centros privados).

Tabla 2

Edad del profesorado adscrito al programa bilingüe

\begin{tabular}{lcccccc}
\hline & \multicolumn{2}{c}{ Centros Públicos } & \multicolumn{2}{c}{ Centros Privados } & \multicolumn{2}{c}{ TOTAL } \\
\cline { 2 - 8 } & Frecuencia & $\%$ & Frecuencia & $\%$ & Frecuencia & $\%$ \\
\hline 20 a 30 & 13 & 21 & 6 & 35 & 19 & 24 \\
\hline 31 a 40 & 22 & 36 & 9 & 53 & 31 & 40 \\
\hline 41 a 50 & 19 & 31 & 2 & 12 & 21 & 27 \\
\hline 51 a 60 & 5 & 8 & 0 & 0 & 5 & 6 \\
\hline 61 hacia delante & 2 & 3 & 0 & 0 & 2 & 3 \\
\hline Total & 61 & 100 & 17 & 100 & 78 & 100 \\
\hline
\end{tabular}

Del mismo modo, el tiempo de ejercicio profesional también es muy diferente entre maestros de centro públicos y maestros de centros privados. Como se puede comprobar en la tabla 3, la mayoría de los maestros de la muestra de centros privados llevan menos de 11 años de ejercicio profesional, estando el porcentaje más alto concentrado en la primera franja (entre 1 y 5 años), y no hay nadie con más de 20 años de anti- 
güedad en la docencia. Sin embargo, en el caso de centros públicos, la mayoría de los maestros de la muestra se sitúan entre 6 y 20 años de experiencia, contando con casos de todas las franjas de antigüedad.

Tabla 3

Tiempo de ejercicio profesional en la enseñanza

\begin{tabular}{lllllll}
\hline & \multicolumn{2}{l}{ Centros Públicos } & \multicolumn{2}{l}{ Centros Privados } & Total \\
\cline { 2 - 7 } & Frecuencia & $\%$ & Frecuencia & $\%$ & Frecuencia & $\%$ \\
\hline 1 a 5 años & 7 & 11 & 7 & 41 & 14 & 18 \\
\hline 6 a 10 años & 18 & 30 & 6 & 35 & 24 & 31 \\
\hline 11 a 15 años & 13 & 21 & 2 & 12 & 15 & 19 \\
\hline 16 a 20 años & 10 & 16 & 2 & 12 & 12 & 15 \\
\hline 21 a 25 años & 5 & 8 & 0 & 0 & 5 & 7 \\
\hline 26 a 30 años & 4 & 7 & 0 & 0 & 4 & 5 \\
\hline 31 a 35 años & 4 & 7 & 0 & 0 & 4 & 5 \\
\hline Total & 61 & & 17 & & 78 & 100 \\
\hline
\end{tabular}

Por último, la tabla 4 presenta algunos datos más específicos sobre la docencia que desempeñan los maestros de esta muestra en el momento en que se realizó la recogida de datos, comprobando como el $58 \%$ de educadores, imparte docencia únicamente en inglés y el $42 \%$ de los maestros imparte enseñanza tanto en castellano como en lengua inglesa (con pocas diferencias entre centros públicos o privados).

Tabla 4

Tipo de docencia

\begin{tabular}{|c|c|c|c|c|c|c|}
\hline & \multicolumn{2}{|c|}{ Centros Públicos } & \multicolumn{2}{|c|}{ Centros Privados } & \multicolumn{2}{|l|}{ Maestros } \\
\hline & Frecuencia & $\%$ & Frecuencia & $\%$ & Frecuencia & $\%$ \\
\hline Docencia en inglés & 36 & 59 & 9 & 53 & 45 & 58 \\
\hline $\begin{array}{l}\text { Docencia en } \\
\text { castellano e inglés }\end{array}$ & 25 & 41 & 8 & 47 & 33 & 42 \\
\hline Total & 61 & & 17 & & 78 & 100 \\
\hline
\end{tabular}

De esta forma, se ha procedido a buscar una representatividad no tanto en términos estadísticos como en términos fenomenológicos, pretendiendo que la diversidad existente en estos Programas esté presente en la muestra de informantes.

El procedimiento de recogida de información mediante la entrevista siguió las siguientes fases: a.- se contactó con el centro para informarles 
e invitarles a participar en la investigación; b.- se informó al profesorado del objetivo de la entrevista y confidencialidad de los datos; c.- se acordaron días y fechas para las entrevistas, siempre de forma directa con la presencia de una investigadora, se grabaron y transcribieron para su posterior análisis.

Atendiendo al criterio temático, la información fue agrupada en torno a categorías, que pueden considerarse como un mapa de significados que reflejan fielmente el contenido de las entrevistas y permite interpretar las valoraciones de los docentes.

\section{Resultados}

De acuerdo con el proceso de categorización seguido, se puede afirmar que las valoraciones de los docentes implicados en las primeras promociones del Programa Bilingüe en la Región de Murcia destacan los siguientes aspectos: satisfacción profesional, apoyo de la Consejería de Educación, coordinación docente, estrategias de enseñanza y resultados del alumnado.

A continuación, se presentan las aportaciones más significativas de la información recopilada en cada una de estas categorías.

\subsection{Satisfacción profesional}

Todos los docentes entrevistados manifiestan estar satisfechos o muy satisfechos con su participación en los programas bilingües. Entre sus declaraciones, destacan aquellas en las que plantean estar muy satisfechos pues les gusta impartir docencia en este Programa y perciben que su vida profesional ha mejorado tras su incorporación al mismo: "Muy satisfecha tengo una vida profesional muy diferente a la de hace cuatro años, nada que ver, los frutos que estoy obteniendo son maravillosos" (E57); "Estoy muy satisfecha en cuanto al trabajo de los compañeros y el mío y el apoyo entre nosotros" (E18); "Muy satisfecha porque he mejorado muchísimo como maestra, tengo más recursos porque me he formado, los resultados son muy buenos y me enorgullece ir luchando día a día con los niños y lograr los objetivos" (E45).

Además, expresan su satisfacción con el ejercicio de su profesión en el Programa Bilingüe aunque eso supone realizar grandes esfuerzos y de- 
dicar parte de su tiempo libre para contribuir a una enseñanza bilingüe de calidad: "Satisfecho porque al ser mi primer año me estoy adaptando a este proyecto, aprendiendo que estoy aprendiendo muchísimo, creo que el camino es bueno y positivo y me gusta" (E54); "Con mi trabajo estoy satisfecha porque la conciencia la tengo tranquila de que no paro de trabajar y mis compañeras también, tienen interés e intentan hacerlo lo mejor posible" (E5).

En coherencia con estas afirmaciones, la práctica totalidad del profesorado entrevistado (93\% en centros públicos y la totalidad en centros educativos privados), manifiesta su deseo de participar en el Programa Bilingüe toda su vida profesional, porque le encanta impartir docencia en lengua inglesa mediante esta metodología: "Muchísimos años, siempre ya que estoy muy motivada con el programa bilingüe" (E3); "Voy a participar siempre. Nunca me he planteado salirme porque me gusta lo que hago" (E4). Incluso los profesores que no tienen plaza definitiva en el centro en el que están en ese momento manifiestan su interés por impartir docencia en la enseñanza bilingüe: "Este año estoy aquí el año que viene no lo sé, pero me gustaría que me dieran un colegio bilingüe porque es un reto a nivel personal" (E8); "Todavía no tengo plaza definitiva, estoy a la deriva, pero el Programa Bilingüe me gusta y si pudiera elegiría enseñanza bilingüe siempre" (E19).

Tan sólo un pequeño número de profesores de centros educativos públicos expresa su incertidumbre acerca del tiempo que va a seguir participando en el Programa Bilingüe, bien por insatisfacción con la implementación del Programa, bien por inquietudes personales y profesionales: "Me apetece seguir en el programa, me gusta lo que hago y diría siempre, pero si es cierto que a veces me canso porque creo que no se puede llevar a cabo el programa de esta forma" (E11).

\subsection{Apoyo de la Consejería de Educación}

Es de destacar que la mayoría del profesorado entrevistado (78\%), con un porcentaje significativamente superior en colegios públicos, afirma estar poco o nada satisfecho con el apoyo de la Consejería de Educación a la implementación del Programa Bilingüe en los centros.

Estos docentes se sienten abandonados por la Administración Educativa, pues no solo reclaman recursos materiales y económicos, así como otros aspectos que parece ser que se prometieron y no se efectuaron posterior- 
mente, sino también atención y preocupación por la implementación de su labor docente ante este nuevo reto educativo: "Yo, al igual que otros maestros pues me consta que algunos piensan como yo, estoy muy descontenta, muy insatisfecha con el funcionamiento del Programa Bilingüe, falta material, incentivos, reconocimiento, ayuda, dinero, apoyo de profesores, auxiliares de conversación... Además, no se están cumpliendo cosas que nos prometieron" (E11); "Con el funcionamiento del programa por la Consejería considero que hay muchas cosas que van a salto de mata, muchos aspectos que los van solucionando sobre la marcha sin haber planteado un proyecto a largo plazo claro" (E60); "Nada satisfecha con el apoyo de la Consejería, nos lanzaron y queda muy bonito tantos colegios bilingües y sin prestarles una atención adecuada. Estoy desmoralizada" (E46).

\subsection{Coordinación docente}

La mayoría del profesorado entrevistado (76\%) declara mantener contacto profesional con maestros de otros colegios adscritos al Programa Bilingüe y conocer la implementación de la enseñanza bilingüe en otros centros. Sin embargo, en este caso las diferencias entre docentes de centros públicos y docentes de centros privados son importantes, como se puede comprobar en la tabla 5.

Tabla 5

Coordinación con maestros de otros centros

\begin{tabular}{lcccccc}
\hline & \multicolumn{3}{c}{ Centros Públicos Centros Privados } & \multicolumn{2}{c}{ Total } \\
\cline { 2 - 7 } & Frecuencia & $\%$ & Frecuencia & $\%$ & Frecuencia & $\%$ \\
\hline No & 12 & 20 & 7 & 41 & 19 & 24 \\
\hline Sí más de 5 maestros & 31 & 51 & & & 31 & 40 \\
\hline Sí menos de 5 maestros & 18 & 29 & 10 & 59 & 28 & 36 \\
\hline Total & 61 & 100 & 17 & 100 & 78 & 100 \\
\hline
\end{tabular}

Son mayoría los docentes de centros educativos públicos que afirman comunicarse con más de 5 maestros de distintos colegios y poseer un gran conocimiento del desarrollo de la enseñanza bilingüe en otros centros. Sin embargo, un considerable porcentaje de docentes de centros privados $(41 \%)$ manifiesta no mantener contacto profesional con docentes de otros centros ni conocer la implementación de la enseñanza bilingüe en otros colegios. 
Por otra parte, la gran mayoría de profesores (88\%) afirma coordinarse con el resto de maestros participantes en el Programa Bilingüe de su colegio. Los docentes de centros educativos públicos expresan reunirse con sus compañeros implicados en la docencia bilingüe con una periodicidad mensual o bien cada mes y medio /dos meses: "Una vez al mes, aunque no es obligatorio, hemos decidido reunirnos todos los maestros del Programa Bilingüe para ver las necesidades de material, nos hemos puesto de acuerdo sobre cuándo le vamos a exigir la parte escrita a los niños, en primero se les pide que reconozcan palabras, en segundo que escriban palabras y pequeñas frases y en tercero realizar pequeños textos. Por ello, conforme ha ido subiendo el programa hemos visto la necesidad de ponernos de acuerdo sobre unos cuantos aspectos porque sino unos maestros exigen una cosas y otros otras y al final es un caos" (E10), "Con todos los maestros del Programa cada dos meses tenemos una reunión en la que intercambiamos ideas, proponemos cosas que mejoraríamos, relatamos experiencias..." (E26).

Siendo pocos los maestros que manifiestan coordinarse con el resto de docentes que imparten áreas a través de la lengua inglesa con una periodicidad mayor: "Con todos los maestros del programa al principio de curso dijimos semanalmente pero como es imposible pues quincenalmente" (E78), o los que afirman reunirse sólo de forma trimestral: "Con todos los de inglés implicados en el programa bilingüe me coordino trimestralmente" (E50).

En cualquier caso, todos los docentes, sin apenas diferencias entre centros públicos y privados, manifiestan que la coordinación con el resto de compañeros adscritos al Programa bilingüe es muy útil al propiciar el intercambio de opiniones, dudas y experiencias sobre tareas y materiales; el trabajo conjunto entre los diferentes grupos de alumnos de un mismo curso ya que todos tienen que seguir la misma línea de actuación; así como la reflexión de la práctica docente y el enriquecimiento profesional.

Sin embargo, la práctica totalidad del profesorado entrevistado (exceptuando un maestro de un centro privado) afirma no poseer una hora semanal de su horario lectivo dedicada a la coordinación con otros compañeros participantes en el Programa Bilingüe y consideran necesario disponer de dicho periodo de tiempo. 


\subsection{Cambio metodológico}

La mayoría del profesorado entrevistado (88\%), sin apenas diferencias entre tipología de centros, afirma que la adscripción a la enseñanza bilingüe ha conllevado un cambio en las estrategias empleadas para facilitar la comprensión y asimilación de los contenidos académicos, ya que se enseñan los contenidos académicos de manera más lúdica, experimental, manipulativa y visual.

A los alumnos se les concede un rol más activo y participativo, fruto del aprendizaje por descubrimiento, al tener que descubrir ellos mismos su entorno observando y manipulando los objetos. Asimismo, estos profesores consideran que este cambio metodológico conlleva beneficios para los estudiantes ya que el aprendizaje es más efectivo: "Ahora es más manipulativa y visual que antes. Science lo aprenden mejor porque es más tangible en inglés que en castellano, porque en castellano abre el libro por la página tal y mira la fotografía del libro, pero aquí si estás dando rugoso y suave te tienes que traer el estropajo y la cinta de raso para que ellos mismos lo comprueben. Es otro mundo" (E46).

Además, expresan que este cambio metodológico ha propiciado que los alumnos sean más conscientes de su aprendizaje, conociendo en cada momento lo que están realizando y el motivo por el que lo están haciendo. Ello ha conllevado que los profesores de la enseñanza bilingüe no solo se preocupen por los contenidos académicos a enseñar a los estudiantes sino por el cómo hacerles llegar esos conocimientos, de ahí la importancia de buscar las mejores técnicas que faciliten esta tarea: "Ahora hay que prestarle atención tanto al contenido como al cómo hacerles llegar el contenido y trabajar dos áreas a la misma vez. Por eso, se ha hecho más complejo y difícil de abordar la enseñanza" (E49).

Las técnicas que se han incorporado en la práctica docente con la introducción de la lengua inglesa en la enseñanza de algunas áreas son las siguientes:

- Utilizan recursos visuales que no empleaban antes, principalmente vídeos, para las explicaciones de contenidos académicos complejos: "...al empezar el Programa necesitas el apoyo visual para que los niños comprendan los contenidos. Y te das cuenta que también puedes utilizar ese apoyo en otras áreas en español. Así pues ahora se aprovecha lo aprendido en bilingüe en otras enseñanzas" (E10). 
- Afirman que ahora utilizan habitualmente la pizarra digital. Estos profesores expresan que este recurso es imprescindible en la enseñanza bilingüe ya que mejora la comprensión de los conocimientos a los estudiantes.

- Y, en menor medida, manifiestan que las técnicas empleadas ahora difieren de las utilizadas antes de su incorporación a la enseñanza bilingüe ya que emplean experimentos, que permitan a los alumnos manipular, observar y tocar materiales para comprender mejor los contenidos académicos; se apoyan más en el lenguaje no verbal, corporal y gestual y recurren al uso de materiales reales, es decir, de objetos cotidianos que tienen en su contexto.

Tan sólo un pequeño número de maestros afirma que, si bien existe el cambio metodológico, éste no ha sido propiciado por la incorporación a la enseñanza bilingüe: "Podría ser porque ahora la enseñanza se presta más a ser más manipulativa pero antes del Programa también podías hacerlo todo más manipulativamente. No creo que en sí por estar en un Programa Bilingüe cambie, porque puedes estar en el bilingüe y seguir igual" (E30).

\subsection{Mejoras en los resultados de los estudiantes}

Todo el profesorado entrevistado muestra estar satisfecho con los logros que los alumnos alcanzan en el Programa Bilingüe. Sin embargo, el grado de satisfacción difiere entre el profesorado de centros públicos y el de centros privados. Así, el $88 \%$ de los maestros de centros privados (frente al 59 en centros públicos) manifiesta estar muy satisfecho con los resultados obtenidos por los alumnos en el programa bilingüe: "Con los alumnos muy satisfecho porque se pueden apreciar diferencias enormes entre alumnos que están en bilingüe y aquellos de cursos superiores que no están en bilingüe" (E67).

Mientras que el $41 \%$ de maestros en centros educativos públicos (frente al $12 \%$ de centros privados o concertados), manifiesta estar solo satisfecho con el rendimiento académico de los estudiantes en esta enseñanza bilingüe: "Con los logros de los alumnos en general satisfecha porque el que tiene capacidad bien pero el que no es apoyado mal pues hay niños que les cuesta aunque se esfuerzan por mejorar y aprender" (E5). 
La totalidad de los docentes entrevistados afirma que los resultados académicos en el área de Lengua Extranjera: inglés de los alumnos adscritos a Programas Bilingües son superiores a los obtenidos por los estudiantes no participantes en la enseñanza bilingüe. Además, consideran mayoritariamente que la motivación de los alumnos por el aprendizaje de la lengua inglesa se ha incrementado significativamente con la enseñanza bilingüe. El interés de los alumnos adscritos en Programas Bilingües por conocer y estudiar inglés ha aumentado y ello ha derivado en una mayor implicación y participación en las actividades diarias realizadas en las clases en las que el inglés se utiliza como lengua vehicular de los contenidos.

Sin embargo, estos docentes enuncian que los resultados académicos de los alumnos adscritos a Programas Bilingües en el área de Conocimiento del Medio son similares a los obtenidos por los estudiantes no participantes en la enseñanza bilingüe. Y la gran mayoría de los entrevistados (91\%), con un porcentaje significativamente superior en centros educativos públicos, manifiesta que la enseñanza bilingüe dificulta el aprendizaje de contenidos a los alumnos con necesidades específicas de apoyo educativo o con inferior nivel académico. Estos docentes enuncian que dependiendo del tupo y grado de deficiencia de los estudiantes las dificultades se agrandan en mayor o menor medida: "El Programa Bilingüe dificulta muchísimo a los niños con necesidades específicas de apoyo educativo y por mucho que reciban algún apoyo en clase al ser inglés siguen teniendo problemas para avanzar en la enseñanza bilingüe" (E15).

\subsection{Propuestas que contribuirían a un mayor éxito del programa bilingüe}

A pesar del grado de satisfacción del profesorado entrevistado, consideran que hay un listado de aspectos que sin lugar a dudas contribuirían a un mayor éxito en la implementación de este Programa en las aulas (ver Tabla 6). 
Tabla 6

Propuestas para un mayor éxito en la enseñanza bilingüe

\begin{tabular}{lccccccc}
\hline & \multicolumn{2}{c}{ Centros } & \multicolumn{2}{c}{ Centros } & \multicolumn{2}{c}{ Total } \\
& Públicos & \multicolumn{2}{c}{ Privados } & & \\
\cline { 2 - 8 } & Frecuencia & $\%$ & Frecuencia & $\%$ & Frecuencia & $\%$ \\
\hline Coordinación & 37 & 61 & 3 & 18 & 40 & 51 \\
\hline Formación & 31 & 51 & 5 & 29 & 36 & 46 \\
\hline Aumentar horas auxiliar & 33 & 54 & & & 33 & 42 \\
\hline Valorar y escuchar docentes & 20 & 33 & 3 & 18 & 23 & 30 \\
\hline Aumentar apoyos inglés & 18 & 30 & 2 & 12 & 20 & 26 \\
\hline Reducción horaria & 27 & 44 & 4 & 24 & 31 & 40 \\
\hline Recursos económicos & 6 & 10 & 12 & 71 & 18 & 23 \\
\hline Recursos espaciales & 4 & 7 & & & 4 & 5 \\
\hline Recursos materiales & 7 & 12 & 1 & 6 & 8 & 10 \\
\hline Intercambio materiales & 5 & 8 & & & 5 & 7 \\
\hline Recursos digitales & 2 & 3 & & & 2 & 3 \\
\hline Coordinación con auxiliar & 7 & 12 & & & 7 & 9 \\
\hline Implicación Consejería & 6 & 10 & & & 6 & 8 \\
\hline Reconocimiento & 4 & 7 & & & 4 & 5 \\
\hline Aumentar reducción & 2 & 3 & & & 2 & 3 \\
coordinador & 61 & 100 & 17 & 100 & 78 & 100 \\
\hline Total & & & & & &
\end{tabular}

El $61 \%$ de maestros en centros educativos públicos (frente al 18\% en centros privados), afirma que se debería de contemplar en su horario lectivo horas destinadas a la coordinación con maestros participantes en el Programa Bilingüe con el fin de tratar temáticas comunes, tomar decisiones y pedir orientaciones a otros docentes con mayor experiencia en la enseñanza bilingüe: "Lo único que me gustaría tener tiempo de coordinación para ir más tranquila, aunque sea con los maestros del mismo nivel y ciclo para tratar temas, porque a veces me agobio cuando tengo una duda y no puede hablar con esa maestra para resolverla" (E45); "Los maestros no tenemos tiempo para programar y juntarnos y así no se puede llevar a cabo un Programa Bilingüe" (E46).

Un poco más de la mitad de docentes del sector público enuncia que se deberían de aumentar el horario de los auxiliares de conversación con el fin de que puedan permanecer más tiempo con cada grupo de alumnos, pues creen que una sesión con los asistentes nativos es insuficiente: "Más horas con el auxiliar de conversación porque una hora 
semanal es como si no tuviésemos nada. Es una lástima que se gasten ese dinero y no se aproveche" (E47).

Casi la mitad de los docentes manifiesta la relevancia de incrementar los recursos ofrecidos a los participantes en el Programa Bilingüe. Realizando un análisis detallado de estos datos, en los colegios privados se menciona la necesidad de aumentar los recursos económicos aportados a cada centro adscrito a la enseñanza bilingüe para la contratación de auxiliares de conversación, o en su defecto, la concesión por parte de la Consejería en materia educativa de este recurso igual que al resto de colegios públicos. Estos docentes no entienden el motivo de su exclusión en un recurso tan esencial como es el ayudante lingüístico: "Nosotros no sabemos dónde ha invertido el dinero en idiomas en los últimos quince años ni cómo, pero no lo vemos. No tenemos auxiliares de conversación por ser centro concertado, sólo nos han dado una placa" (E63).

Continuando con la concreción de los recursos, algunos maestros destacan que se deberían ofrecer más recursos materiales, tales como vídeos sobre diversos contenidos académicos de Conocimiento del Medio adaptados a niños de Educación Primaria, así como libros en inglés para la biblioteca de aula. Se plantean también la necesidad de poseer mejores recursos espaciales, reclamando tener aulas más grandes para poder realizar ciertas actividades que requieren ciertos agrupamientos y laboratorios o aulas bilingües para la realización de experimentos, argumentado que el área de Conocimiento del Medio es muy manipulativa.

Un pequeño grupo de profesores del sector público declara la necesidad de un mayor intercambio de experiencias y recursos materiales con docentes pertenecientes al Programa Bilingüe mediante jornadas o un banco de datos en el que se recopile todo el material proporcionada por cada uno de los maestros acerca de diversas temáticas. Estos educadores resaltan la importancia de intercambiar materiales pues enuncian la dificultad de encontrar vídeos acerca de ciertas temáticas adaptados a niños. Además, estos maestros destacan la necesidad de una mayor unión y coordinación entre centros educativos adscritos a la enseñanza bilingüe no solo a nivel regional, sino local, con el fin de intercambiar materiales, opiniones y ayudarse unos a otros en aquellos aspectos que presenten dificultad para algunos docentes: "Mucha más coordinación entre centros. Esto lo echo mucho de menos pues hay ideas buenas que se están llevando a cabo en otros centros y es una pena que no las conozcamos, cosas que hago yo pueden venir bien a otros y al revés" (E53). 
El $46 \%$ de educadores, con un porcentaje mayor en centros educativos públicos (51\% frente al 29\% del sector privado), enuncia que sería imprescindible aumentar la formación del profesorado participante en el Programa Bilingüe, no sólo a los maestros con destino definitivo en colegios bilingües, sino también al resto de maestros que se inician en la enseñanza bilingüe. Los docentes argumentan que los maestros interinos tienen el mismo derecho a recibir formación que el resto de profesorado definitivo en un centro bilingüe. Además, manifiestan la importancia de recibir formación sobre la enseñanza bilingüe pues al ir incrementado el número de colegios bilingües seguramente sean destinados a otros centros participantes en el Programa: "Es necesario ofrecer más formación a los maestros que se van a incorporar al Programa Bilingüe y mayor formación continua para los docentes que estamos implicados en el mismo" (E12); "Habría que preparar a todo el personal docente, interinos y no interinos, porque si tenemos que enfrentarnos a una clase bilingüe qué menos que sepamos a qué atenernos para estar a la altura de las circunstancias" (E67).

El $40 \%$ de los maestros, con un porcentaje mayor en colegios públicos, declara ser fundamental contemplar en su horario lectivo un periodo de tiempo para la preparación de materiales, pues resaltan que dedican mucho de su tiempo libre a la realización de esta tarea: "Horas para preparar materiales y actividades, falta mucho tiempo. Sobre todo tiempo para organizarme y realizar actividades que me gustaría hacerlas y no las hago por no tener tiempo para prepararlas" (E47).

Los docentes entrevistados manifiestan la importancia de que la Consejería de Educación escuche su opinión acerca de la implementación del Programa Bilingüe y de los aspectos que se podrían mejorar para contribuir a un mayor éxito de la enseñanza bilingüe. Ellos afirman ser los principales conocedores de las necesidades reales del Programa Bilingüe, fruto de su día a día en las aulas con los alumnos. Asimismo, estos profesores resaltan la necesidad de sentirse apoyados y valorados por la Administración Educativa, de percibir su implicación y compromiso por esta enseñanza bilingüe, pues afirman haber pedido orientación sobre algún aspecto complejo o dificultad a este organismo y no han tenido respuesta. Es por ello que perciben que el Programa Bilingüe se desarrolla actualmente por su interés, su dedicación y su entrega hacia esta enseñanza: "Lo más urgente es que estamos en el Programa Bilingüe pero estamos abandonados, no me siento cuidada, ni mimada ni 
protegida. Estamos luchando, nosotros los profesores, porque queremos que esto siga adelante pero realmente no vemos apoyo de la Consejería" (E39).

El 26\% de los educadores, con un porcentaje más elevado en el sector público, afirma que se debería incrementar el número de maestros especialistas en inglés en todos los centros educativos, para ofrecer más apoyos a los estudiantes que necesitan ese refuerzo educativo de un docente: "Más maestros de inglés en los centros porque los apoyos de lengua y matemáticas los puede realizar cualquier maestro, pero los de inglés solo los puede llevar a cabo otro de inglés" (E46).

Con porcentajes en torno al 10\%, los docentes del sector público no solo manifiesta la necesidad de incrementar las horas del ayudante lingüístico por grupo de estudiantes, sino también de contemplar un periodo de tiempo de su horario lectivo para la coordinación con estos asistentes nativos; así como de obtener implicación por parte de la Administración Educativa antes de incrementar el número de colegios pertenecientes a la enseñanza bilingüe. Estos docentes manifiestan su malestar con la Consejería de Educación por el aumento significativo año tras año de colegios bilingües, pues resaltan que en lugar de ampliar la enseñanza bilingüe todos los años debería preocuparse por la implementación del Programa Bilingüe y realizar un seguimiento de los centros pertenecientes a esta enseñanza.

\section{Discusión y conclusiones}

A pesar de las quejas generalizadas del profesorado participante en la enseñanza bilingüe por la falta de implicación y colaboración de la Consejería competente en materia educativa con el Programa Bilingüe, la valoración de los maestros sobre la enseñanza a través de una segunda lengua es satisfactoria, corroborando así resultados de otros estudios (Infante, Benvenuto, \& Lastrucci, 2009; Lorenzo, Casal, Moore y Alfonso, 2009; Fernández \& Halbach, 2011; Hunt, Neofitou y Redford, 2009; Méndez \& Pavón, 2012; Sasajima, 2013; Ferre, Jódar y Martín, 2014; De Matos, 2014; García, 2014; Pladevall-Ballester, 2015).

Los maestros están contentos con los resultados académicos de los estudiantes, ya que perciben su mejora de las habilidades lingüísticas de inglés. El entusiasmo y la retroalimentación de los alumnos recibida por 
los docentes, es uno de los factores que los impulsa a continuar implicándose en el Programa Bilingüe. La participación de los maestros en la enseñanza bilingüe ha derivado en un aumento de su motivación por su labor docente considerando su trabajo actual más interesante. La incorporación al Programa Bilingüe ha contribuido a un crecimiento profesional de los maestros (de acuerdo con los resultados hallados también por Dobson, Pérez y Johnstone, 2011; Fernández \& Halbach, 2011 y De Matos, 2014), ya que la enseñanza a través de una segunda lengua les ha permitido conocer y enriquecerse de otros enfoques pedagógicos y metodológicos y, en definitiva, mejorar su labor docente. Asimismo, los docentes afirman su satisfacción con la metodología de esta enseñanza bilingüe y no se imaginan utilizando anteriores procesos de enseñanza, por lo que, como expresa Roldán (2011), los maestros han adquirido elementos positivos a implantar de forma permanente en sus prácticas docentes.

Los resultados relativos a la continuidad de la Educación Primaria en la enseñanza bilingüe constatan lo expresado por Pladevall-Ballester (2015), acerca del deseo generalizado de los maestros implicados en los Programas Bilingües de seguir impartiendo docencia en estos Programas toda su vida profesional. En esta línea, cabe destacar la incertidumbre de algunos docentes acerca de su participación en la enseñanza bilingüe ya que el trabajo en el Programa Bilingüe resulta agotador. $Y$ es que como afirman Fernández \& Halbach (2011), el escaso apoyo e implicación de la Administración educativa es uno de los factores conducentes al agotamiento de estos maestros.

Para finalizar, se presentan algunas de las propuestas percibidas por los maestros participantes en el Programa Bilingüe que contribuirían a un mayor éxito del mismo, pues creemos que la necesidad de la participación de los docentes, principales conocedores de los beneficios y perjuicios del Programa Bilingüe, en la toma de decisiones sobre aspectos concernientes a la enseñanza bilingüe, pueden ayudar a que estos Programas funcionen mejor en la totalidad de los centros donde se están desarrollando.

Los docentes entrevistados sienten que la implementación de estos Programas recae fundamentalmente en su voluntariedad. Así, enfatizan la importancia de sentirse apoyados, valorados y reconocidos por su labor docente en estos Programas por parte de la Administración educativa. Más concretamente, estos docentes demandan una mayor implicación 
de la Consejería competente en educación en aspectos tales como: incremento de recursos o actualización de los recursos tecnológicos existentes, mayor número de auxiliares de conversación y, sobre todo, conceder un tiempo de coordinación con ellos y establecer un tiempo para la coordinación con otros maestros implicados en el Programa Bilingüe.

Otra de las mejoras a implantar por los profesores participantes en el Programa Bilingüe es la realización de un seguimiento de los centros educativos adscritos a esta enseñanza, con el propósito de conocer la implementación del Programa en cada uno de ellos. Asimismo, los resultados constatan la necesidad de priorizar la consolidación de los Programas Bilingües que están implementados sobre la ampliación desmesurada de la enseñanza bilingüe a otros colegios.

La necesidad de incrementar el personal docente especialista en inglés en los centros educativos para realizar apoyos y, a su vez, beneficiar a los estudiantes que precisan ese refuerzo para lograr una comprensión eficaz de los contenidos, como exponen otros estudios, es otra de las demandas imprescindibles de estos maestros.

\section{Referencias}

De Matos, M.E.E. (2014). CLIL as a Catalyst for Developing Reflective Practice in Foreign Language Teacher Education (Tesis Doctoral). Universidad de Porto, Portugal.

Dobson, A., Pérez, M.D. y Johnstone, R. (2011). Programa de Educación Bilingüe en España. Informe de evaluación. Resultados de la investigación independiente sobre el Programa de Educación Bilingüe del Ministerio de Educación y el British Council en España. Ministerio de Educación y British Council.

Ferre, F.M., Jódar, F.J. y Martín, J. (2014). El programa bilingüe de alemán en la Enseñanza Secundaria: un ejemplo de coordinación y cooperación didáctica. Magazin/Extra, 1, 26-32. Recuperado de: http://www.fage.es/magazin_extra/magazin_e_1/26a32.pdf Fernández, R. \&Halbach, A. (2011).Analysing the Situation of Teachers in the Madrid Bilingual Project after Four Years of Implementation. In Y. Ruiz de Zarobe, J. M. Sierra y F. Gallardo del Puerto (Eds.), Content and Foreign Language Integrated Learning. Contributions to Multilingualism in European Contexts (pp. 241-270). Frankfurt am Main, Alemania: Peter Lang

García, E. (2014). Diseño, desarrollo y evaluación de los programas de enseñanza bilingüe en el entorno escolar de Soria (Tesis doctoral). Universidad de Valladolid, España.

Halbach, A. (2008). Una metodología para la enseñanza bilingüe en la etapa de Primaria. Revista de Educación, 346, 455-466.

Hunt, M., Neofitou, A. \& Redford, J. (2009). Developing CLIL training for modern languages teacher trainees. In D. Marsh, P. Mehisto, D. Wolff, R. Aliaga, T. Asikainen, M.J. 
Frigols-Martin, S. Hughes \& G. Langé (Eds.), CLIL Practice: Perspectives from the Field (pp. 110-116). Jyväskylä, Finlandia: University of Jyväskylä.

Infante, D., Benvenuto, G. \&Lastrucci, E. (2009). The Effects of CLIL from the Perspective of Experienced Teachers.In D. Marsh, P. Mehisto, D. Wolff, R. Aliaga, T. Asikainen, M.J. Frigols-Martin, S. Hughes \& G. Langé (Eds.), CLIL Practice: Perspectives from the Field (pp. 156-163). Jyväskylä, Finlandia: University of Jyväskylä.

Kvale, S. (2011). Las entrevistas en investigación cualitativa. Madrid: Morata.

Lorenzo, F., Casal, S., Moore, P. y Alfonso, Y.M. (2009). Bilingüismo y Educación: Situación de la Red de Centros Bilingües en Andalucía. Sevilla, España: Fundación Centro de Estudios Andaluces.

Méndez, M.C. \& Pavón, V. (2012). Investigating the coexistence of the mother tongue and the foreign language through teacher collaboration in CLIL contexts: perceptions and practice of the teachers involved in the plurilingual programme in Andalusia. International Journal of Bilingual Education and Bilingualism, 15(5), 573-592.

Nussbaum, L. (1999). Perspectives actuals per al'ensenyamentil'aprenentatge de Ilengües estrangeres a l'educatió obligatòria. Perspectiva Escolar, 232, 2-8.

Pavón, V. \& Rubio, F.D. (2010).Teacher's s Concerns and Uncertainties about the Introduction of CLIL Programmes.Porta Linguarum: Revista Internacional de Didáctica de las lenguas extranjeras, 14, 45-58.

Pladevall-Ballester, E. (2015). Exploring primary school CLIL perceptions in Catalonia: students', teachers' and parents' opinions and expectations.International Journal of Bilingual Education and Bilingualism, 18(1), 45-59.

Roldán, H. (2011). Promoción del bilingüismo mediante la inclusión del inglés en un modelo pedagógico enfocado hacia el aprendizaje autónomo. Voces y Silencios: Revista Latinoamericana de Educación, 2(2), 111-127.

Salkind, N. J. (2009). Exploring research (7th ed.). Upper Saddle River, NJ: Pearson

Sasajima, S. (2013). How CLIL Can Impact on EFL Teachers' Mindsets about Teaching and Learning: An Exploratory Study on Teacher Cognition. International CLIL Research Journal, 2(1), 55-66.

Siguán, M. y Mackey, W.F. (1986).Educación y Bilingüismo. Madrid: Santillana.

Tójar, J.C. (2006). Investigación cualitativa: comprender y actuar. Madrid: La Muralla. 
